\title{
Local excision in mid-to-low rectal cancer patients who revealed clinically total or near-total regression after preoperative chemoradiotherapy; a proposed trial
}

Jong Lyul Lee ${ }^{1 \dagger}$, Seok-Byung Lim" ${ }^{1 \dagger}$, Chang Sik Yu ${ }^{1 *}$, In Ja Park', Yong Sik Yoon', Chan Wook Kim', Seong Ho Park², Jong Seok Lee ${ }^{2}$, Yong Sang Hong ${ }^{3}$, Sun Young Kim³ ${ }^{3}$ Jeong Eun Kim³, Jong Hoon Kim², Jin-hong Park ${ }^{4}$,

Jihun $\mathrm{Kim}^{5}$ and Minkyu Han ${ }^{6}$

\begin{abstract}
Background: Preoperative chemoradiotherapy (pre-CRT) followed by total mesorectal excision (TME) is currently a standard therapy for locally advanced mid-to-low rectal cancer. Less aggressive, organ-preserving option such as local excision (LE) or watchful wait can alternatively be used for patients who respond well to pre-CRT. Highresolution rectal magnetic resonance imaging (MRI) is one of the most useful methods to assess pre-CRT response, and the MERCURY group has shown that the MR tumor regression grade (mrTRG) correlated with the pathologic TRG. The aim of this study is to compare postoperative complication and oncologic outcomes between LE and TME in mid-to-low rectal cancer patients whose tumors are MrTRG grade 1 (radiological complete remission) or 2 (predominant fibrosis; near-complete remission) after pre-CRT.
\end{abstract}

Methods: A prospective, double-arm, randomized, open-labeled, single center, clinical trial will be conducted in patients with mid-to-low rectal cancer whose tumors are mrTRG 1/2 after pre-CRT at the Asan Medical Center, Seoul, Korea, after approval from the Institution Review Board. Patient medical records will be de-identified using a serial number to protect personal information. Inclusion criteria will include rectal adenocarcinoma with an inferior border $<8 \mathrm{~cm}$ from the anal verge, mrTRG 1/2, age $>20$, and provision of informed consent. Postoperative complications will be assessed by Clavien-Dindo Classification Grade. Oncologic and functional outcomes will be collected and risk factors related to these outcomes will be investigated.

Discussion: We believed that the rate of postoperative complication of LE will be comparable to that of TME in mid-to-low advanced rectal cancer patients with a favorable response after pre-CRT.

Trial registration: KCT0002579 (https://cris.nih.go.kr) Dec-2017.

Keywords: Rectal cancer, Preoperative chemoradiotherapy, Regression, Postoperative complication, Local excision, Total mesorectal excision

\footnotetext{
* Correspondence: csyu@amc.seoul

Jong Lyul Lee and Seok-Byung Lim are co-lead investigators on this study. ${ }^{1}$ Division of Colon and Rectal Surgery, Department of Surgery, University of Ulsan College of Medicine, Asan Medical Center, 88, Olympic-ro 43-gil,

Songpa-gu, Seoul 05505, Korea

Full list of author information is available at the end of the article
}

(c) The Author(s). 2019 Open Access This article is distributed under the terms of the Creative Commons Attribution 4.0 International License (http://creativecommons.org/licenses/by/4.0/), which permits unrestricted use, distribution, and reproduction in any medium, provided you give appropriate credit to the original author(s) and the source, provide a link to the Creative Commons license, and indicate if changes were made. The Creative Commons Public Domain Dedication waiver (http://creativecommons.org/publicdomain/zero/1.0/) applies to the data made available in this article, unless otherwise stated. 


\section{Background}

In the treatment of rectal cancer, total mesorectal excision (TME) improves oncologic outcomes and adding preoperative chemo-radiotherapy (pre-CRT) to TME has been shown to effectively improve local control [14]. For this reason, pre-CRT followed by TME is recommended for patients with locally advanced mid-to-low rectal cancer [5-9]. However, significant complications are associated with TME, including anastomotic leak, sexual and urinary dysfunction, and frequent stool passage, on top of the peri-operative morbidity associated with all major surgery [10-12]. In addition, abdominoperineal resection (APR) involves a permanent stoma and low anterior resection (LAR) often involves at least a temporary stoma [8, 12, 13]. The decision of a surgical strategy must take oncologic outcomes, postoperative complications, and quality of life into account.

For patients with favorable responses to pre-CRT, organ-preserving strategies such as local excision (LE) and watchful waiting are becoming more accepted [1419]. There has been some debate regarding the oncologic safety of LE following pre-CRT in advanced mid-to-low rectal cancer [14-18]. LE following pre-CRT for advanced rectal cancer could be a particularly good option for patients showing a complete pathologic response and achieving tumor regression not only in the primary tumor but also in the mesorectal metastatic lymph nodes [20-24]. Although the tumor regression grade (TRG) obtained using high-resolution rectal magnetic resonance imaging (mrTRG), as proposed by the MERCURY study group, correlated with the pathologic TRG [20, 21, 23, 24], mrTRG did not reflect the pathologic T status. A recent study from our institution showed that a status of mrTRG1 predicted a pathologic complete remission (pCR) with $60.6 \%$ accuracy and the projected completion TME rate of mrTRG1 was $25.6 \%$. This results suggests that LE, compared with watchful wait, is more likely to result in pCR [25].

There have been several reports on the feasibility of performing LE following pre-CRT, most of which are focused on either clinical T2-3 rectal cancer or on cases of pCR [26-28]. However, the oncologic safety of LE in patients with a pathologic early $\mathrm{T}$ status after pre-CRT has not been established [29]. Therefore, data on long term outcomes with respect to the pathologic status and the type of surgery in patients with locally advanced rectal cancer is needed.

\section{Methods}

\section{Objective}

The purpose of the current study is to determine whether LE in mid-to-low rectal cancer patients with
mrTRG 1 or 2 tumors after pre-CRT is safe and effective in terms of surgical and oncologic outcomes.

\section{Primary outcome measures}

All complications within 30 days of the surgical intervention will be assessed using the Clavien-Dindo Classification Grade.

\section{Secondary outcomes measures}

Secondary outcomes will be as follows:

1. Oncologic outcomes: 3-year disease-free survival (\% DFS), 3-year overall survival (\% OS), and recurrence rate (\%).

2. Functional outcomes: quality of life, as measured using EORTC QLQ-C30 (version 3) and rectal function, as measured using manometry, low anterior resection syndrome (LARS) score, and the Wexner Fecal Incontinence Score.

3. The rate of permanent stoma formation (\%).

4. Factors affecting complete remission and the accuracy of mrTRG.

5. Oncologic outcome in patient subgroups.

\section{Study design}

A prospective, double-arm, randomized, open-labeled, single center, clinical trial was initiated at Asan Medical Center, Seoul, Korea beginning in July 2017. (Fig. 1).

\section{Study population}

Patients who were diagnosed with pathologically confirmed rectal adenocarcinoma $<8 \mathrm{~cm}$ from the anal verge with clinically $\mathrm{T} 3$ or $\mathrm{N}+$ before pre-CRT and an mrTRG grade 1 or 2 based on post-CRT images will be eligible for the study when they meet the following inclusion criteria:

- Male and female patients between the age of 20 and 80;

- Good performance status (ECOG performance status <2);

- Capable of providing informed consent.

\section{Exclusion criteria}

- Rectal cancer $>8 \mathrm{~cm}$ from the anal verge;

- Microperforated lesion before pre-CRT;

- Concurrent metastasis at a diagnosis;

- Synchronous colorectal cancer;

- Previous history of radiotherapy;

- Other synchronous or metachronous malignancies diagnosed within 5 years of the diagnosis of rectal cancer; 


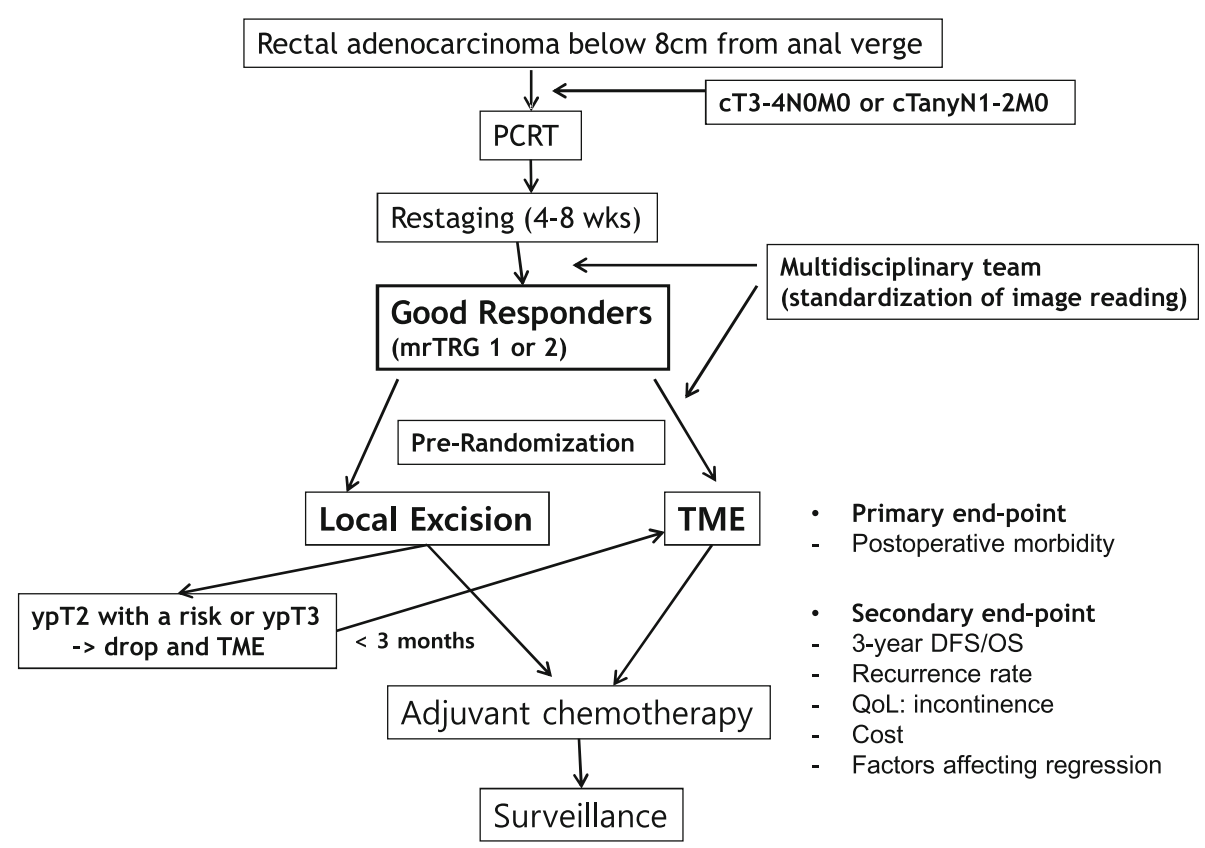

Fig. 1 Study design including primary and secondary study endpoints

- Enrollment in other clinical trials except for those of adjuvant therapies;

- Pregnant or breastfeeding women.

\section{Pre-registration procedures \\ Preoperative chemoradiotherapy}

Pre-CRT was delivered at $50.4 \mathrm{~Gy}$ to the primary tumor and the pelvis in 25 fractions. Concurrent chemotherapy during radiotherapy consisted of 5 -fluorouracil (5-FU; $375 \mathrm{mg} / \mathrm{m}^{2} /$ day) and leucovorin $\left(\mathrm{LV} ; 20 \mathrm{mg} / \mathrm{m}^{2} /\right.$ day $)$ or capecitabine $\left(825 \mathrm{mg} / \mathrm{m}^{2}\right) .5$-FU and LV were given on three consecutive days for two courses during the first and fifth weeks of radiotherapy, while capecitabine was given twice daily during the entire radiotherapy period without a weekend break.

\section{Preoperative restaging}

For assessing the pre-CRT response, digital rectal examination, sigmoidoscopy, abdomino-pelvic computed tomography $(\mathrm{CT})$, chest $\mathrm{CT}$, trans-rectal ultrasonography and MRI were performed within 2 weeks before surgery. Two board-certified abdominal radiologists independently assessed the response to CRT on post-CRT MRI using mrTRG (1-5) according to published definitions: 1 , complete radiologic response; 2 , dense fibrosis with no obvious residual tumor; $3,>50 \%$ fibrosis or mucin with visible residual tumor; 4, small areas of fibrosis or mucin but mostly tumor, or tumor growth; 5, same appearance as baseline. The enrollment of a study cohort requires a multidisciplinary team including a colorectal surgeon, medical oncologist, radiation oncologist, and radiologist.

\section{Treatment strategies}

\section{Standard care of the control arm}

TME is the standard treatment and is undertaken 6-8 weeks after the completion of pre-CRT. All operations were performed by experienced colorectal surgeons (> 100 rectal cancer cases per year). Temporary diverting ileostomy is occasionally performed in cases of a very low anastomotic level with considerable tension, air leak, or severe comorbidities. We do not perform lateral lymph node dissection routinely.

\section{Investigational treatment of the experimental arm}

LE will be performed via conventional transanal excision or transanal minimal invasive surgery. During LE, remnant scar tissue will be excised with at least a $1 \mathrm{~cm}$ resection margin. Patients who have poorly differentiated or mucinous ypT2 tumors with lympho-vascular invasion, perineural invasion, tumor budding, and close resection margins $(<1 \mathrm{~mm})$ will be recommend for TME within 3 months. If the patient undergoes TME, he or she will be evaluated as part of the TME group. If the patient does not choose to undergo TME, he or she will be excluded from the study and followed-up for additional subgroup analysis.

\section{Postoperative follow-up schedule}

Patients will be followed for 3-year after surgery. Follow-up will consist of a physical examination and 
blood tests, including carcinoembryonic antigen (CEA) levels every 6 months after surgery. Chest X-ray and abdomino-pelvic CT every 6 months after surgery, chest CT will be performed every 6-12 months, and colonoscopy performed at 6-12 months and 24-36 months after surgery (Table 1).

\section{Primary outcome}

The primary endpoint of the current study is the rate of postoperative complications. Postoperative events occurring up to 30 days after surgery will be evaluated by the Clavien-Dindo Classification, any events grade 2 or higher will be considered postoperative complications.

\section{Secondary study endpoints}

1. Disease-free survival rate (\%), overall survival rate (\%) and recurrence rate (\%) at 3 years.

2. Functional outcomes, including quality of life (EORTC-E030) and score of fecal incontinence (Wexner's score) at 6,12 and 24 months after surgery.

3. Rate of permanent stoma formation (\%) at 3 years.

4. Accuracy of mrTRG (positive predictive value and negative predictive value).

\section{Sample size calculation}

The sample size was calculated based on two-sided non-inferiority test with for $80 \%$ power and a significant level of 0.05 , one-sided. We assume that the rate of postoperative complications on day 30 will be $15 \%$ in the TME group and $15 \%$ in the LE group, with a non-inferiority margin of $15 \%$. Based on these calculations, a total of 67 patients should be allocated to each group; assuming a 5\% dropout rate, we plan to enroll 140 patients. The 15\% rate of postoperative complications cited above is based on published findings [26-28, 30].

\section{Randomization and data management}

After checking the response of pre-CRT, the participants with mrTRG 1 or 2 tumor will be assigned using random-number table with sealed envelopes by an external research worker who control the data. According to mrTRG 1 or 2 , the allocation sequence will be stratified. The external research worker will collect the data and a statistician who provided the sample size calculation and data analysis plan will be analyze the data. The data entry, coding, security and storage with backup will be processed by the external research worker and simultaneously the statistician will check the accuracy and the value of the data. After finishing the collection of data, the external research worker, the statistician will access the dataset and investigators will access after analyses of the dataset.

\section{Data analysis}

The intent-to-treat (ITT) analysis set will include all subjects who are randomized into a surgery group. The modified ITT analysis set will include all subjects who undergo pre-CRT. The per protocol (PP) analysis set will

Table 1 Follow-up schedule and study procedure for study cohort

\begin{tabular}{|c|c|c|c|c|}
\hline Documentation & Restaging & LE vs. TME & Visit 1 & Visit 2-6 \\
\hline Study procedures & Screening & Surgery & Checking complications & Routine checkup \\
\hline Period after pre-CRT & 4-8 weeks & $4-8$ weeks & $8-16$ weeks & Every 6 months after Op. \\
\hline Getting permission & $\bullet$ & & & \\
\hline Screening & $\bullet$ & & & \\
\hline Assigning serial number & $\bullet$ & & & \\
\hline Demographics & $\bullet$ & & & \\
\hline Physical exam & $\bullet$ & & $\bullet$ & $\bullet$ \\
\hline Heart/ lung evaluation & $\bullet$ & & & \\
\hline Chest CT & $\bullet$ & & & - (Selective) \\
\hline APCT & $\bullet$ & & & $\bullet$ \\
\hline MR, rectal cancer & $\bullet$ & & & - (Selective) \\
\hline Endoscopy & $\bullet$ & & & - (Selective) \\
\hline Laboratory test & $\bullet$ & & $\bullet$ & $\bullet$ \\
\hline Endorectal sonography & $\bullet$ & & & \\
\hline Manometry/questionnaire & $\bullet$ & & & - (Selective) \\
\hline Checking complications & & & $\bullet$ & $\bullet$ \\
\hline Selection of surgery type & & $\bullet$ & & \\
\hline
\end{tabular}

Pre-CRT Preoperative chemoradiotherapy, Op., Operation, LE Local excision, TME Total mesorectal excision, CT Computed tomography, APCT Abdomino-pelvic CT, MR Magnetic resonance 
include only the subjects in the ITT group who complete the study protocol. Patients who withdraw informed consent or who develop serious adverse events will be excluded from the PP analysis set. The modified ITT set will be used for analysis of primary and secondary study endpoints. The PP analysis set will be used for subgroup analysis. Baseline characteristics will also be analyzed in the ITT group.

Variables and outcomes will be analyzed using chisquare tests (for proportions) or Fischer's exact test, as appropriate. Continuous data will be analyzed using Student's t-test or Mann-Whitney U tests, as appropriate. Cumulative risk rates will be calculated using the Kaplan-Meier method and compared using log-rank tests. Multivariate analyses using binary logistic regression will be used to assess the risk of postoperative complications. Statistical significance will be defined as $p<0.05$, and all analyses will be performed using SPSS software, version 22 (IBM Corporation, Armonk, NY, USA).

\section{Safety}

The main outcome will be the detection and documentation of postoperative complications. The severity of complications will be classified according to the Clavien-Dindo Classification Grade and will be registered in the case reporting form. Currently, the oncologic outcome of pre-CRT followed by LE is considered to be equivalent to that of pre-CRT followed by TME for patients who respond well to pre-CRT. If the pathologic results show ypT2 with risks, ypT3 or ypT4, surgeons will recommend to perform TME for oncologic safety of the participants. The current study will meet with all local legal requirements and meet all requirements of the ICH Guideline for Clinical Safety Data Management, Definition and Standards for Expedited Reporting, Topic E2A.

\section{Discussion}

Although pre-CRT followed by TME has become the standard treatment for patients with locally advanced rectal cancer, recent evidences suggests that organ-preserving strategies such as LE and watchful waiting can be used as an alternatively to TME in patients with a favorable response to pre-CRT without compromising oncologic outcomes and high rate of major LARS [14-19, 31]. Numerous tools can be used to assess the tumor response after pre-CRT, including physical examination, endorectal ultrasound, endoscopy, and MRI. MR staging is currently considered one of the most useful methods of evaluating the response to pre-CRT [20, 24, 25]. However, the accuracy rate of mrTRG for predicting PCR is only approaximately $60 \%$ [25]. Considering that ypT status reflects the ypStage and that patients with pCR or ypT1N0M0 have a more favorable prognosis [29], accurate examination of tumor status is needed.
Recently, our institution reported a retrospective analysis demonstrating that oncologic outcomes of pre-CRT followed by LE were comparable to those of TME, even in patients with lymph node involvement [27]. A recent meta-analysis also suggested that there are no differences in local recurrence rates, disease-free survival, and overall survival between rectal cancer patients that received LE or TME [28]. In patients with early-stage rectal cancer, several studies found that LE did not compromise the oncologic outcome but did result in organ preservation [28]. Based on previous studies, if lymph nodes are positive after pre-CRT, patients are optimally treated with TME with regional lymph node clearance, in line with the current standard of treatment [29], but a patient that achieves a complete or near-complete response after pre-CRT can consider LE.

In terms of postoperative complications, only one patient $(2.1 \%)$ in our previous study developed a postoperative complication (perianal fistula) that required prolonged hospitalization [27]. In a recent prospective study of LE in patients with T2 rectal cancer, surgery-related adverse events above grade 3 occurred in 16 patients (21\%) and grade 1 or 2 complications occurred in 50 patients (65\%) [26]. Although complications of LE after pre-CRT are generally less severe than complications of TME, the rate of overall complications including dehiscence of the rectal suture line resulted from anal pain are higher than TME after pre-CRT [26]. Based on the assumption that the oncologic outcomes of LE and TME are similar for patients with a favorable response (mrTRG 1 or 2) after pre-CRT, further prospective studies on surgical outcomes in LE after pre-CRT are needed.

Pre-CRT followed by LE causes minimal loss of anorectal function and quality of life, similar to watchful waiting [26]. TME results in postoperative loss of anorectal, urinary and sexual function, and also carries the risk of permanent stoma formation [10]. In mid-to-low advanced rectal cancer, the use of pre-CRT has decreased the rate of permanent stoma formation; however, in our recent study, approximately $30 \%$ of patients receiving TME after pre-CRT did develop a permanent stoma [27]. A recent prospective study suggested that pre-CRT followed by LE might be an alternative to TME for patients with rectal cancer who have a favorable response to pre-CRT or who seek organ preservation because the Fecal Incontinence Severity Index (FISI) score and the Functional Assessment of Cancer Therapy-Colorectal (FACT-C) scores did not substantially decrease after surgery [26].

Previous studies, even prospective studies for LE or watchful waiting, suffer from the lack of standardized selection criteria. Recently, the MECURY group convened multidisciplinary teams (MDT) to decide which patients should defer surgery and which patients should receive 
standard treatemtn after pre-CRT [22]. Our institution also reported that case discussions in MDT meetings resulted in altered clinical decisions in $>10 \%$ cases for advanced and recurrent colorectal cancer [32]. Based on this experience, in the current study, specialized faculty, including a colorectal surgeon, radiologist, medical oncologist, radiation oncologist, gastroenterologist, and pathologist, will select the study patients that show a favorable response after pre-CRT. The data on postoperative complications in LE and TME after pre-CRT collected through the current study will provide valuable information about the risk factors of LE after pre-CRT.

This prospective study has some weaknesses because this study will be performed based on the real practice in our institution. Recent studies insists that the interval between completion of pre-CRT and the assessment of tumor response is increased up to 12 weeks and this longer interval might increase the rate of pathologic CR $[33,34]$. On the other hand, in this prospective study, TME is undertaken $6-8$ weeks after the completion of pre-CRT according to the real practice in our institution. The schedule of restaging is designed within 2 weeks before surgery considering waiting time of MRI in our institution and minimizing dropouts, however, almost patients have restaged at 2 or 3 days before surgery on the real practice. About 10\% of lymph node infiltration and the $15 \%$ of local recurrence in the ypT2 were worrisome for this study $[27,29,35]$. Nevertheless, recent reports showed the overall survival was not significantly different between the LE and TME groups [27, 36, 37]. The evidences of LE for ypT2 tumors are still lack, therefore, our study determines the effect of LE in ypT2 tumors on oncologic outcomes.

\section{Abbreviations}

APR: Abdominoperineal resection; CEA: Carcinoembryonic antigen;

CT: Computed tomography; DFS: Disease-free survival; ITT: Intent-to-treat; LE: Local excision; MDT: Multidisciplinary team; MRI: Magnetic resonance imaging; mrTRG: MRI_tumor regression grade; mrTRG1: Radiologically complete remission; mrTRG2: Essentially compete fibrosis; OS: Overall survival; pCR: Pathologic complete remission; PP: Per protocol; Pre-CRT: Preoperative chemoradiotherapy; TME: Total mesorectal excision; TRG: Tumor regression grade; WHO: World Health Organization

\section{Acknowledgements}

Not Applicable.

\section{Funding}

This study is funded by a grant (2017-0239) from the Asan Institute for Life Sciences, Asan Medical Center, Seoul, Republic of Korea. The funder will not relate to the study process including study design; collection, management, analysis and interpretation of data; writing of the report; submission for publication; authorship.

\section{Availability of data and materials}

Not applicable in this study protocol. After finishing the enrollments of the participants, the raw datasets are available from the corresponding author on reasonable request.

\section{Authors' contributions}

$J L L$ made substantial contributions to the study conception and design, drafting critical revision of the manuscript, and approval of the final version to be submitted. S-BL made substantial contributions to the study conception and design, drafting critical revision of the manuscript, and approval of the final version to be submitted. JLL and S-BL are co-lead investigators on this study. CSY made substantial contributions to the study conception and design, acquisition, drafting critical revision of the manuscript, and approval of the final version to be submitted as the corresponding author. IJP made some contributions to study design, helped to the manuscript, and gave approval of the final version to be submitted. YSY made some contributions to study design, helped to the manuscript, and gave approval of the final version to be submitted. CWK made some contributions to study design, helped to the manuscript, and gave approval of the final version to be submitted. SHP made some contributions to study design, helped to the manuscript, and gave approval of the final version to be submitted. JSL made some contributions to study design, helped to the manuscript, and gave approval of the final version to be submitted. YSH made some contributions to study design, helped to the manuscript, and gave approval of the final version to be submitted. SYK made some contributions to study design, helped to the manuscript, and gave approval of the final version to be submitted. JEK made some contributions to study design, helped to the manuscript, and gave approval of the final version to be submitted. JHK made some contributions to study design, helped to the manuscript, and gave approval of the final version to be submitted. JP made some contributions to study design, helped to the manuscript, and gave approval of the final version to be submitted. JK made some contributions to study design, helped to the manuscript, and gave approval of the final version to be submitted. $\mathrm{MH}$ made some contributions to study design, provided data from his patients, helped to the manuscript, and gave approval of the final version to be submitted.

\section{Ethics approval and consent to participate}

The study protocol was approved by the Institutional Review Board of Asan Medical Center (reference number: 2017-0239), and the study was performed in accordance with the Declaration of Helsinki. All of the participants will give their written consent to participate.

\section{Consent for publication}

Not Applicable in this study protocol.

\section{Competing interests}

The authors declare that they have no conflict of interest.

\section{Publisher's Note}

Springer Nature remains neutral with regard to jurisdictional claims in published maps and institutional affiliations.

\section{Author details}

'Division of Colon and Rectal Surgery, Department of Surgery, University of Ulsan College of Medicine, Asan Medical Center, 88, Olympic-ro 43-gil, Songpa-gu, Seoul 05505, Korea. ${ }^{2}$ Department of Radiology, University of Ulsan College of Medicine, Asan Medical Center, 88, Olympic-ro 43-gil, Songpa-gu, Seoul 05505, Korea. ${ }^{3}$ Department of Medical Oncology, University of Ulsan College of Medicine, Asan Medical Center, 88, Olympic-ro 43-gil, Songpa-gu, Seoul 05505, Korea. ${ }^{4}$ Department of Radiation Oncology, University of Ulsan College of Medicine, Asan Medical Center, 88, Olympic-ro 43-gil, Songpa-gu, Seoul 05505, Korea. ${ }^{5}$ Department of Pathology, University of Ulsan College of Medicine, Asan Medical Center, 88, Olympic-ro 43-gil, Songpa-gu, Seoul 05505, Korea. ${ }^{6}$ Department of Clinical Epidemiology and Biostatistics, University of Ulsan College of Medicine, Asan Medical Center, 88, Olympic-ro 43-gil, Songpa-gu, Seoul 05505, Korea.

Received: 27 August 2018 Accepted: 4 April 2019

Published online: 29 April 2019

\section{References}

1. Heald R, Ryall R. Recurrence and survival after total mesorectal excision for rectal cancer. Lancet. 1986;327(8496):1479-82. 
2. Syk E, Glimelius B, Nilsson P. Factors influencing local failure in rectal cancer: analysis of 2315 patients from a population-based series. Dis Colon Rectum. 2010;53(5):744-52.

3. Rahbari NN, Ulrich AB, Bruckner T, Münter M, Nickles A, Contin P, Löffler T, Reissfelder C, Koch M, Büchler MW. Surgery for locally recurrent rectal cancer in the era of total mesorectal excision: is there still a chance for cure? Ann Surg. 2011;253(3):522-33.

4. van Gijn W, Marijnen CA, Nagtegaal ID, Kranenbarg EM-K, Putter H, Wiggers T, Rutten HJ, Påhlman L, Glimelius B, van de Velde CJ. Preoperative radiotherapy combined with total mesorectal excision for resectable rectal cancer: 12-year follow-up of the multicentre, randomised controlled TME trial. Lancet Oncol. 2011;12(6):575-82.

5. Gérard J-P, Conroy T, Bonnetain F, Bouché O, Chapet O, Closon-Dejardin MT, Untereiner M, Leduc B, Francois É, Maurel J. Preoperative radiotherapy with or without concurrent fluorouracil and leucovorin in T3-4 rectal cancers: results of FFCD 9203. J Clin Oncol. 2006;24(28):4620-5.

6. Roh MS, Colangelo LH, O'Connell MJ, Yothers G, Deutsch M, Allegra CJ, Kahlenberg MS, Baez-Diaz L, Ursiny CS, Petrelli NJ. Preoperative multimodality therapy improves disease-free survival in patients with carcinoma of the rectum: NSABP R-03. J Clin Oncol. 2009;27(31):5124.

7. Sauer R, Becker H, Hohenberger W, Rödel C, Wittekind C, Fietkau R, Martus P, Tschmelitsch J, Hager E, Hess CF. Preoperative versus postoperative chemoradiotherapy for rectal cancer. N Engl J Med. 2004;351(17):1731-40.

8. Sauer R, Liersch T, Merkel S, Fietkau R, Hohenberger W, Hess C, Becker H, Raab H-R, Villanueva M-T, Witzigmann H. Preoperative versus postoperative chemoradiotherapy for locally advanced rectal cancer: results of the German CAO/ARO/AIO-94 randomized phase III trial after a median followup of 11 years. J Clin Oncol. 2012;30(16):1926-33.

9. Sebag-Montefiore D, Stephens RJ, Steele R, Monson J, Grieve R, Khanna S, Quirke P, Couture J, de Metz C, Myint AS. Preoperative radiotherapy versus selective postoperative chemoradiotherapy in patients with rectal cancer (MRC CR07 and NCIC-CTG C016): a multicentre, randomised trial. Lancet. 2009;373(9666):811-20.

10. Kim KH, Yu CS, Yoon YS, Yoon SN, Lim S-B, Kim JC. Effectiveness of biofeedback therapy in the treatment of anterior resection syndrome after rectal cancer surgery. Dis Colon Rectum. 2011;54(9):1107-13.

11. Lim SB, Yu CS, Hong YS, Kim TW, Kim JH, Kim JC. Long-term outcomes in patients with locally advanced rectal cancer treated with preoperative chemoradiation followed by curative surgical resection. J Surg Oncol. 2012; 106(6):659-66

12. Lim SB, Yu C, Kim C, Yoon Y, Park I, Kim J. Late anastomotic leakage after low anterior resection in rectal cancer patients: clinical characteristics and predisposing factors. Color Dis. 2016;18(4):0135-40.

13. Park J, Yoon SM, Yu CS, Kim JH, Kim TW, Kim JC. Randomized phase 3 trial comparing preoperative and postoperative chemoradiotherapy with capecitabine for locally advanced rectal cancer. Cancer. 2011;117(16):3703-12.

14. Creavin B, Ryan E, Martin S, Hanly A, O'Connell P, Sheahan K, Winter D. Organ preservation with local excision or active surveillance following chemoradiotherapy for rectal cancer. Br J Cancer. 2017;116(2):169.

15. Rullier E, Rouanet P, Tuech J-J, Valverde A, Lelong B, Rivoire M, Faucheron J$L$, Jafari M, Portier G, Meunier B. Organ preservation for rectal cancer (GRECCAR 2): a prospective, randomised, open-label, multicentre, phase 3 trial. Lancet. 2017;390(10093):469-79.

16. Renehan AG, Malcomson L, Emsley R, Gollins S, Maw A, Myint AS, Rooney PS, Susnerwala S, Blower A, Saunders MP. Watch-and-wait approach versus surgical resection after chemoradiotherapy for patients with rectal cancer (the OnCoRe project): a propensity-score matched cohort analysis. Lancet Oncol. 2016;17(2):174-83.

17. Pucciarelli S, De Paoli A, Guerrieri M, La Torre G, Maretto I, De Marchi F, Mantello G, Gambacorta MA, Canzonieri V, Nitti D. Local excision after preoperative chemoradiotherapy for rectal cancer: results of a multicenter phase II clinical trial. Dis Colon Rectum. 2013;56(12):1349-56.

18. Perez RO, Habr-Gama A, Lynn PB, São Julião GP, Bianchi R, Proscurshim I, GamaRodrigues J. Transanal endoscopic microsurgery for residual rectal cancer (ypT0-2) following neoadjuvant chemoradiation therapy: another word of caution. Dis Colon Rectum. 2013;56(1):6-13.

19. Habr-Gama A, Perez RO, Nadalin W, Sabbaga J, Ribeiro U Jr, e Sousa AHS Jr, Campos FG, Kiss DR, Gama-Rodrigues J. Operative versus nonoperative treatment for stage 0 distal rectal cancer following chemoradiation therapy: long-term results. Ann Surg. 2004;240(4):711.

20. Patel UB, Taylor F, Blomqvist L, George C, Evans H, Tekkis P, Quirke P, SebagMontefiore D, Moran B, Heald R. Magnetic resonance imaging-detected tumor response for locally advanced rectal cancer predicts survival outcomes: MERCURY experience. J Clin Oncol. 2011;29(28):3753-60.

21. Bhoday J, Smith F, Siddiqui MR, Balyasnikova S, Swift RI, Perez R, Habr-Gama A, Brown $G$. Magnetic resonance tumor regression grade and residual mucosal abnormality as predictors for pathological complete response in rectal cancer postneoadjuvant chemoradiotherapy. Dis Colon Rectum. 2016; 59(10):925-33.

22. Battersby NJ, Dattani M, Rao S, Cunningham D, Tait D, Adams R, Moran BJ, Khakoo S, Tekkis P, Rasheed S. A rectal cancer feasibility study with an embedded phase III trial design assessing magnetic resonance tumour regression grade (mrTRG) as a novel biomarker to stratify management by good and poor response to chemoradiotherapy (TRIGGER): study protocol for a randomised controlled trial. Trials. 2017;18(1):394

23. Sclafani F, Brown G, Cunningham D, Wotherspoon A, Mendes LST, Balyasnikova S, Evans J, Peckitt C, Begum R, Tait D. Comparison between $\mathrm{MRI}$ and pathology in the assessment of tumour regression grade in rectal cancer. Br J Cancer. 2017;117(10):1478.

24. Battersby NJ, Balyasnikova S, Brown G. Guiding post-treatment decisions in rectal cancer: mrTRG is a practical place to start. Oncology. 2014;28(8):677.

25. Jang J, Lee J, Park S, Park H, Park I, Kim J, Choi S, Kim J, Yu C, Kim J. Magnetic resonance tumour regression grade and pathological correlates in patients with rectal cancer. Br J Surg. 2018;105(12):1671-79.

26. Garcia-Aguilar J, Renfro LA, Chow OS, Shi Q, Carrero XW, Lynn PB, Thomas CR Jr, Chan E, Cataldo PA, Marcet JE. Organ preservation for clinical T2N0 distal rectal cancer using neoadjuvant chemoradiotherapy and local excision (ACOSOG Z6041): results of an open-label, single-arm, multiinstitutional, phase 2 trial. The lancet oncology. 2015;16(15):1537-46.

27. Shin YS, Park J-h, Yoon SM, Kim JC, Yu CS, Lim S-B, Park IJ, Kim TW, Hong YS, Kim KP. Total Mesorectal excision versus local excision after preoperative Chemoradiotherapy in rectal Cancer with lymph node metastasis: a propensity score-matched analysis. Int J Radiat Oncol Biol Phys. 2018;101(3):630-9.

28. Shaikh I, Askari A, Ourû S, Warusavitarne J, Athanasiou T, Faiz O. Oncological outcomes of local excision compared with radical surgery after neoadjuvant chemoradiotherapy for rectal cancer: a systematic review and meta-analysis. Int J Color Dis. 2015;30(1):19-29.

29. Park IJ, You YN, Skibber JM, Rodriguez-Bigas MA, Feig B, Nguyen S, Hu C-Y, Chang GJ. Comparative analysis of lymph node metastases in patients withypT0-2rectal cancers after neoadjuvant chemoradiotherapy. Dis Colon Rectum. 2013;56(2):135.

30. Jang TY, Yu CS, Yoon YS, Lim S-B, Hong S-M, Kim TW, Kim JH, Kim JC. Oncologic outcome after preoperative chemoradiotherapy in patients with pathologic T0 (ypT0) rectal cancer. Dis Colon Rectum. 2012;55(10):1024-31.

31. Smith JD, Ruby JA, Goodman KA, Saltz LB, Guillem JG, Weiser MR, Temple LK, Nash GM, Paty PB. Nonoperative management of rectal cancer with complete clinical response after neoadjuvant therapy. Ann Surg. 2012; 256(6):965-72

32. Jung SM, Hong YS, Kim TW, Park J-h, Kim JH, Park SH, Kim AY, Lim S-B, Lee Y-J, Yu CS. Impact of a multidisciplinary team approach for managing advanced and recurrent colorectal Cancer. World J Surg. 2018;42(7):2227-33.

33. Figueiredo N, Panteleimonitis S, Popeskou S, Cunha JF, Qureshi T, Beets GL, Heald RJ, Parvaiz A. Delaying surgery after neoadjuvant chemoradiotherapy in rectal cancer has no influence in surgical approach or short-term clinical outcomes. Eur J Surg Oncol. 2018;44(4):484-9.

34. Du D, Su Z, Wang D, Liu W, Wei Z. Optimal interval to surgery after neoadjuvant chemoradiotherapy in rectal cancer: a systematic review and meta-analysis. Clin Colorectal Cancer. 2018;17(1):13-24.

35. Stijns RC, de Graaf EJ, Punt CJ, Nagtegaal ID, Nuyttens JJ, van Meerten E, Tanis PJ, de Hingh $I H$, van der Schelling GP, Acherman Y. Long-term oncological and functional outcomes of chemoradiotherapy followed by organ-sparing transanal endoscopic microsurgery for distal rectal cancer: the CARTS study. JAMA Surg. 2019;154(1):47-54.

36. Yang KM, Lim S-B, Lee JL, Kim CW, Yoon YS, Park IJ, Yu CS, Kim JC. Local excision for ypT2 rectal cancer following preoperative chemoradiation therapy: it should not be justified. Int J Color Dis. 2018;33(4):487-91.

37. Lee L, Kelly J, Nassif GJ, Atallah SB, Albert MR, Shridhar R, Monson JR. Chemoradiation and local excision for T2N0 rectal Cancer offers equivalent overall survival compared to standard resection: a national Cancer database analysis. J Gastrointest Surg. 2017;21(10):1666-74. 